\title{
BUILDINGS CHANGE DETECTION BASED ON SHAPE MATCHING FOR MULTI-RESOLUTION REMOTE SENSING IMAGERY
}

\author{
Medbouh Abdessetar, Yanfei Zhong* \\ State Key Laboratory of Information Engineering in Surveying, Mapping and Remote Sensing \\ Wuhan University, 129 Luoyu Road, Wuhan 430079, P.R. China
}

\author{
Commission III, WG III/6
}

KEY WORDS: Buildings change detection, Remote sensing imagery, Multi-resolution images, Shape matching.

\begin{abstract}
:
Buildings change detection has the ability to quantify the temporal effect, on urban area, for urban evolution study or damage assessment in disaster cases. In this context, changes analysis might involve the utilization of the available satellite images with different resolutions for quick responses. In this paper, to avoid using traditional method with image resampling outcomes and saltpepper effect, building change detection based on shape matching is proposed for multi-resolution remote sensing images. Since the object's shape can be extracted from remote sensing imagery and the shapes of corresponding objects in multi-scale images are similar, it is practical for detecting buildings changes in multi-scale imagery using shape analysis. Therefore, the proposed methodology can deal with different pixel size for identifying new and demolished buildings in urban area using geometric properties of objects of interest. After rectifying the desired multi-dates and multi-resolutions images, by image to image registration with optimal RMS value, objects based image classification is performed to extract buildings shape from the images. Next, CentroidCoincident Matching is conducted, on the extracted building shapes, based on the Euclidean distance measurement between shapes centroid (from shape $T_{0}$ to shape $T_{1}$ and vice versa), in order to define corresponding building objects. Then, New and Demolished buildings are identified based on the obtained distances those are greater than RMS value (No match in the same location).
\end{abstract}

\section{INTRODUCTION}

Buildings change detection has many applications in updating geographic information system database, urban evolution estimation and damage assessment in disaster cases. It aims to present automatic analysis of satellite images for urban evolution or risk management where decision-making processes require to have at its disposal reliable and fast support tool that allows a precise monitoring of urban areas.

Moreover, the most commonly used technique for detecting land features is image classification. Currently, due to the increase of satellites sensor resolution, high-resolution imagery offers more details and spectral information about land objects which make it the most appropriate data for studying complex landscape as urban environment. Consequently, traditional image classification algorithms, based on pixels, became useless due to small separability between classes where the classification accuracy drops and the classified output shows a salt-pepper effect which make it difficult to handle the variety of buildings shape and the heterogeneity of roof types (Yonghong et al, 2016).

In this fact, Object-Based Image Analysis (OBIA) can provide a solution to define the objects' shape, by grouping homogenous pixels, as a base element for post-classification change detection process to overcome salt-pepper effect (Lei et al, 2016). Also, in emergency cases, change detection process might use the available high-resolution images with different scales for quick actions where traditional methods fail. In addition, image resampling can influence the accuracy of the final product which leads to imprecise analysis. Despite the availability of many object-based change detection methods for highresolution remote sensing images, few approaches have been developed for multi-scale images that remain a hot topic.

In this context, Feature Vector Analysis (FVA), proposed by Penglin Zhang (Penglin et al, 2012), is a change detection method for multi-resolution remote sensing images. It is based on corresponding buildings shape comparison, by similarity measurement, to identify buildings' shape changes in multitemporal imagery. Note that shape similarity is defined based on contour shape descriptors.

Although shape similarity measurement is used for buildings change detection, new and demolished buildings must be defined for good analysis of change detection results. In this paper, the proposed method is based on shape centroid coincidence matching, to identify new and demolished buildings, via Euclidean distance measurement between multitemporal objects' shape. In view of buildings shape can be extracted from remote sensing images and corresponding objects shapes are similar in multi-scale imagery, shape matching is suitable for change detection using multi-resolution images. It allows comparing the extracted objects' shape without image resampling. This methodology consists of the subsequent steps: (1) Image pre-processing with radiometric and geometric corrections (2) Object-based image classification

* Corresponding author: Yanfei Zhong, State Key Laboratory of Information Engineering in Surveying, Mapping and Remote Sensing, Wuhan University, Hubei Province, China. E-mail: zhongyanfei@whu.edu.cn 
and buildings shape extraction, (3) Centroid-coincident shape matching to identify new and demolished building.

\section{BACKGROUND}

Buildings change detection is a very important topic for many researches those focus on urban area evolution. Thus, traditional pixel-based techniques for change detection are used to quantify objects changes using spectral information of image pixels (reflectance values). A comparative study (Zhang and $\mathrm{Xu}, 2008)$ is established by comparing three methods: Image differencing, Image rationing and post-classification comparison. This study demonstrates that image differencing and image rationing are very easy to implement and they consume few time to produce a change detection map with 'change - no change' information. Also, post classification comparison leads to identify the nature of change by defining the image pixels those change their class over time.

However, the development of sensors technology by increasing the spectral and spatial resolution of remote sensing imagery makes the traditional change detection methods less effective, due to the low separability between pixels' values, which call for the development of new methods. Hence, Object-Based Image Analysis (OBIA) is developed in the last decade to overcome the traditional methods drawbacks. Object-based change detection is used for high-resolution images by segmentation procedure to create image objects as the basic elements for change detection process. It is argued that objectbased change detection is the most reliable method for highresolution images and demonstrates strengths over pixel based method to overcome several problems in change detection process especially reduction of small spurious changes (Gang et al, 2012).

In addition, multi-resolution remote sensing imagery can be used for change detection procedure where the abovementioned methods fail to compare multi-scale images. In this case, image resampling is the available solution to establish change detection map. However, the modification of the original pixel size of an image can lead to a modification of spectral information of that pixel too and produce errors in change detection results (Rene et al, 2012).

Consequently, in order to avoid losing spectral information of image objects, shape extraction and comparison is a useful method for change detection based on objects geometry. This method has the advantage of using geometric properties rather than spectral properties of objects of interest and allows comparing objects, extracted from multi-resolution images, without resampling process to produce a reliable change detection results.

\section{BUILDINGS CHANGE DETECTION BASED ON SHAPE MATCHING FOR MULTI- RESOLUTION REMOTE SENSING IMAGERY}

The proposed approach for buildings change detection, using multi-resolution remote sensing images, is based on buildings extraction from multi-date imagery by object-oriented image classification. Then, buildings shape is used to identify new and demolished buildings based on shape matching.

The flowchart of this approach is shown in the figure bellow:

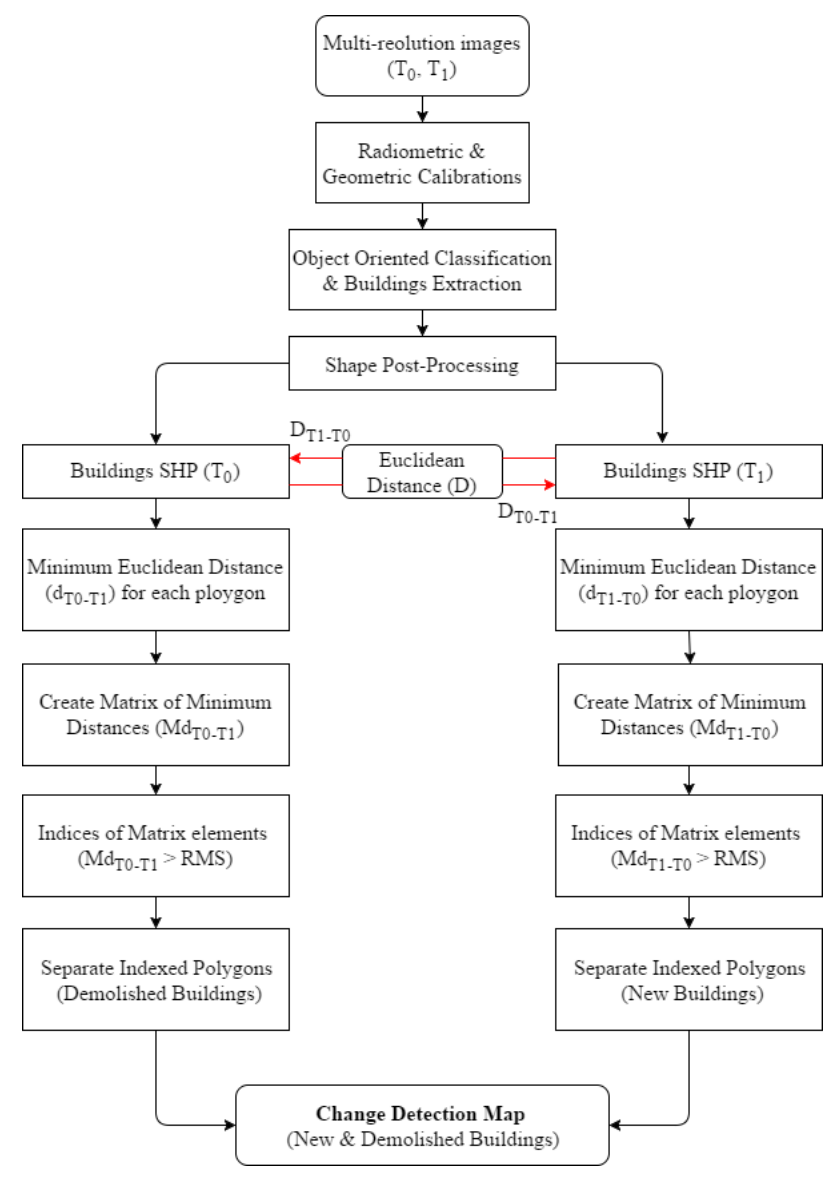

Figure 1. Flowchart of identifying New and Demolished buildings from multi-resolution images by shape matching

\subsection{Image Processing and objects extraction}

After applying radiometric corrections and geometric rectifications using UTM projection to align the images with a suitable average error (RMS) value by ENVI 5.1 software, object-based image classification is conducted by eCognition 9 software in order to extract buildings shape using the classification hierarchy diagram below:

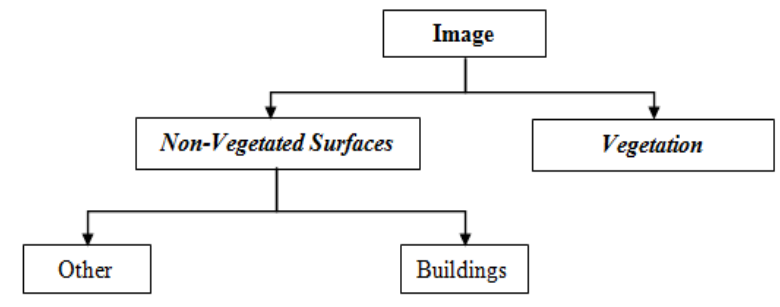

Figure 2. Classification hierarchy for buildings extraction

Based on image segmentation, object-based image classification is the appropriate method to classify high-resolution remote sensing images (Shabnam and Yun, 2013). The classification process is conducted based on user defined class descriptors parameters related to spectral, geometric, contextual and texture information of the obtained image segments to classify the objects by applying a threshold (Elsharkawy et al, 2012). Also, fuzzy logic classification of image objects is used, as a class descriptor, by means of membership functions in order to assign 
the degree of membership of each object to fulfil the given class conditions.

Hence, buildings class descriptor parameters are defined as a knowledge base for buildings classification. Then, the obtained buildings class is exported as shape-file (vector map) which represents the basic element for change detection process.

\subsection{Shape Post-processing}

This process is accomplished using ArcGIS 10 software by realizing the following steps:

\subsubsection{Polygonal approximation}

In order to reduce the segmentation effect, on the obtained buildings shape, polygonal approximation is conducted based on Douglas-Peucker algorithm to minimize the number of points required to represent geometric forms of building polygons. Note that, the approximation degree is related to the tolerance value defined by the user. This algorithm uses pointto-edge distance tolerance. It starts by splitting the polygon into polylines and creating a simplification line joining the endpoints, of each polyline, and calculating the perpendicular distance between this line and the remaining vertices to define their closeness. Then, for each vertex, if the obtained distance is greater than a specified tolerance, the algorithm adds the vertex to the simplified line and uses it as a start point for the next simplification process until adding all vertices with a distance greater than the defined tolerance value.

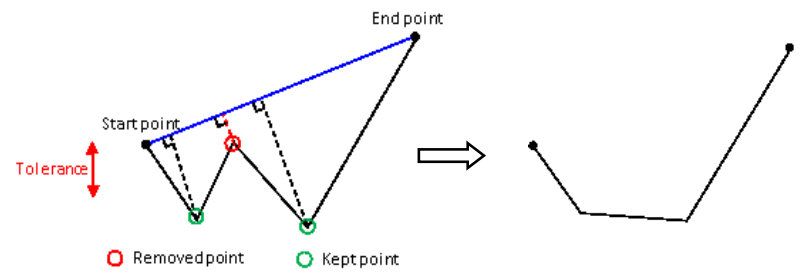

Figure 3. Douglas-Peucker algorithm principal

\subsubsection{Shape attributes definition}

Since the proposed method is based on shape centroids to match corresponding buildings polygons, it is compulsory to define $(\mathrm{x}, \mathrm{y})$ coordinates of each polygon's centroid that express the location of buildings. Note that, the coordinates are defined based on UTM projection.

\subsection{Centroid-coincident matching}

This method based on Euclidean distance (D) measurement between tow polygons' centroid to extract matched shapes:

$$
D=\sqrt{\left(x_{1}-x_{2}\right)^{2}+\left(y_{1}-y_{2}\right)^{2}}
$$

Where: $\quad \mathrm{D}=$ Euclidean distance between polygons centroid $\mathrm{x}_{1}, \mathrm{x}_{2}, \mathrm{y}_{1}, \mathrm{y}_{2}=$ centroid coordinates of polygons.

This technique is used to define corresponding shape geometries in order to perform similarity measurement and define similarity degree between them (Zuoquan and Roger, 2005). In our case, it is performed on the obtained candidate features shape to verify, automatically, the existence of corresponding building features in the same location that leads to identify new and demolished buildings as follow:

- Calculate the Euclidean distance (D) between each polygon of buildings shape $\left(\mathrm{T}_{0}\right)$ and all polygons of buildings shape $\left(T_{1}\right)$ and vice versa;

- Take the minimum distance of the obtained measurement for each polygon $(\mathrm{d}=\operatorname{Min}(\mathrm{D}))$;

- Use the RMS value, obtained from geometric rectification process, as a threshold to verify the existence of corresponding buildings shapes in the same position for unchanged buildings $(\mathrm{d} \leq \mathrm{RMS})$;

- Extract building polygons with distance (d) greater than RMS,

- Define new buildings as the polygons with distances (d), measured from polygons of buildings shape $\left(\mathrm{T}_{0}\right)$ to polygons of buildings shape $\left(\mathrm{T}_{1}\right)$, greater the RMS: dT0-T1 > RMS;

- Define demolished buildings as the polygons with distances, measured from polygons of buildings shape $\left(\mathrm{T}_{1}\right)$ to polygons of building shape $\left(\mathrm{T}_{0}\right)$, greater the RMS: $\mathrm{d}_{\mathrm{T} 1-\mathrm{T} 0}>\mathrm{RMS}$.

Note that, the use of RMS as a threshold means that if we have two points, represents the centroid of two building' polygons, inside a circle with a radius equal to RMS then these polygons occupy the same position in different time (matched pair).

As a result, after realizing the above-described procedures, change detection map is established based on the obtained new and demolished buildings shape.

Therefore, Shape matching is very useful to compare building shapes extracted from multi-date and multi-resolution image without image resampling. It uses geometric properties rather than spectral information to analyze objects changes over time.

\section{RESULTS AND DISCUSSION}

In order to implement the proposed method and verify the feasibility, tow multi-date images with different resolutions are used. These images cover a part of ZengCheng district in Guangzhou city of Guangdong province in China. The first image is acquired by WorldView-2 satellite in 2014 with $0.5 \mathrm{~m}$ resolution and size of $1393 \times 1040$ pixels, while the second image is acquired by Yaogan-24 satellite in 2015 with $1 \mathrm{~m}$ resolution and size of $697 \times 521$ pixels (Figure 4).
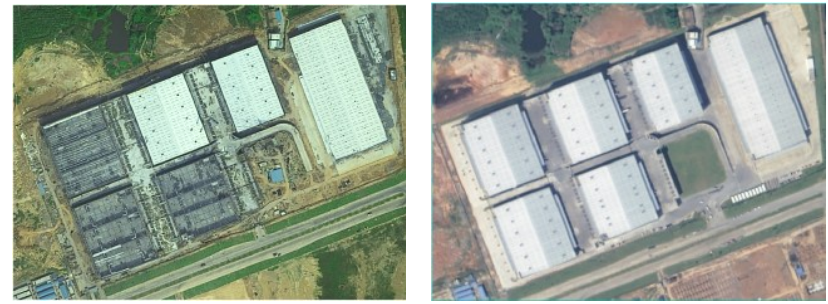

Figure 4. Study area with multi-temporal images. Left: 2014 image $(0.5 \mathrm{~m})$, Right: 2015 image $(1 \mathrm{~m})$ 
Firstly, radiometric correction and geometric rectification are applied to enhance and align the images with an average position error between them RMS $=2.94 \mathrm{~m}$. Then, eCognition software is used to classify the above-described images by object-oriented image classification as shown in Figure 5a and Figure 5b. Thus, buildings shape is exported and enhanced, using ArcGIS software by polygonal approximation, based on the resulted buildings' class as illustrated in Figure $5 \mathrm{c}$ and Figure 5d.

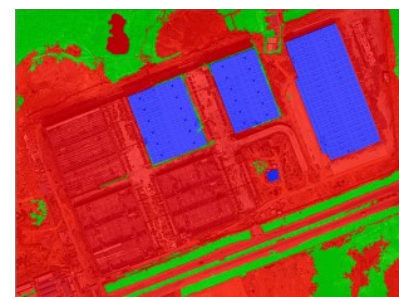

Buildings

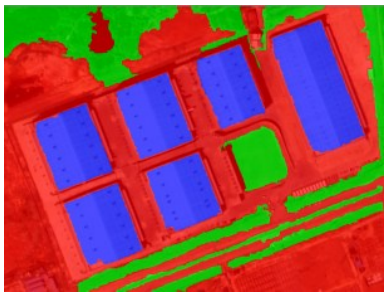

Vegetation

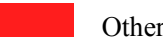

(a) 2014 image classification

(b) 2015 image classification

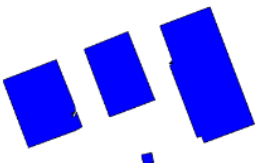

(c) Buildings shape from 2014 image

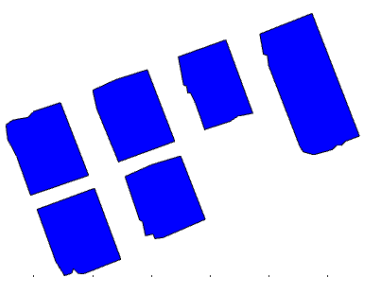

(d) Buildings shape from 2015 image
Figure 5. Object-oriented image classification and buildings extraction

Object-oriented image classification produces the basic element of the proposed buildings change detection approach. In the perspective to use the obtained buildings shape for change detection process, the centroid coordinates $(\mathrm{x}, \mathrm{y})$ are defined for each polygon using ArcGIS software. Subsequently, the table below shows the results of shape matching of the obtained buildings shape from 2014 image and the buildings' shape extracted from 2015 image to identify demolished buildings based on the obtained Euclidean distances and RMS value:

\begin{tabular}{|lll|}
\hline \multicolumn{3}{|c|}{ RMS $=2.94 \mathrm{~m}$} \\
\hline Polygon pairs & Euclidean & Results \\
$\mathrm{ID}\left(\mathrm{T}_{0}, \mathrm{~T}_{1}\right)$ & Distance $(\mathrm{m})$ & \\
\hline 0,2 & 2.25 & Unchanged \\
1,1 & 1.10 & Unchanged \\
2,0 & 0.88 & Unchanged \\
3, non & 142.70 & Demolished \\
\hline
\end{tabular}

Table 1. Corresponding polygon pairs between buildings shape (2014) and buildings shape (2015) to identify Demolished buildings

Also, by defining corresponding polygons pairs between buildings shape obtained from 2015 image and buildings shape obtained from 2014 image, New buildings are identified using Euclidean distance and RMS as shown in Table 2.

\begin{tabular}{|lll|}
\hline \multicolumn{3}{|c|}{ RMS $=2.94 \mathrm{~m}$} \\
\hline $\begin{array}{l}\text { Polygon pairs ID } \\
\left(\mathrm{T}_{1}, \mathrm{~T}_{0}\right)\end{array}$ & $\begin{array}{l}\text { Euclidean } \\
\text { Distance }(\mathrm{m})\end{array}$ & Results \\
\hline 0,2 & 0.88 & Unchanged \\
1,1 & 1.10 & Unchanged \\
2,0 & 2.25 & Unchanged \\
3, non & 157.83 & New \\
4, non & 219.90 & New \\
5, non & 142.70 & New \\
\hline
\end{tabular}

Table 2. Corresponding polygons pairs between buildings shape (2015) and buildings shape (2014) to identify New buildings

Finally, change detection map is produced based on the identified new and demolished buildings polygons as shown in the figure below:

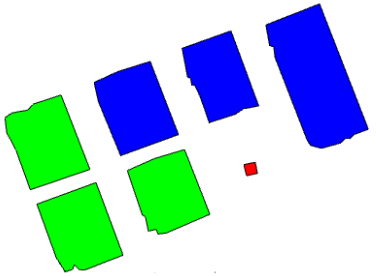

(a) The obtained change detection map

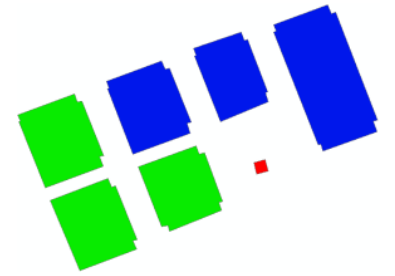

(b) Ground Truth change detection map

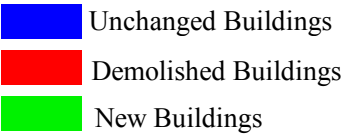

Figure 6. Change detection map with New and Demolished buildings shape

For visual check, the proposed method shows a good performance to identify new and demolished buildings shape (Figure 6a). However, a quantitative analysis of the obtained results is needed in order to verify the accuracy compared to other change detection methods. For this purpose, change detection based on post-classification comparison, by objectoriented image classification, is used. Note that the classification results of the images are obtained after image resampling process.

For the accuracy assessment, the ground truth shape (Figure 6b) is used as a reference data. This shape is obtained by manual digitizing, using ArcGIS software, to create buildings shape of area of interest that reflect the real building objects.

The tables below show the accuracy check of the proposed building change detection method and change detection based on post classification comparison:

\begin{tabular}{|clllllc|}
\hline & \multicolumn{5}{c|}{ Ground truth } \\
\cline { 2 - 6 } & & Changed & Unchanged & Sum & Accuracy & Omission \\
& Changed & 40250 & 2664 & 42914 & $94 \%$ & $6 \%$ \\
Post- & Unchanged & 2350 & 47336 & 49686 & $95 \%$ & $5 \%$ \\
Classification & Sum & 42600 & 50000 & 92600 & \\
comparison & Accuracy & $94 \%$ & $95 \%$ & & & \\
& Omission & $6 \%$ & $5 \%$ & & \\
\cline { 2 - 6 } & Overall accuracy $=94 \%$ & \multicolumn{5}{c}{ Kappa $=0.89$} \\
\hline
\end{tabular}

Table 3. Accuracy of change detection based on postclassification comparison 


\begin{tabular}{|clllllc|}
\hline & \multicolumn{5}{c|}{ Ground truth } \\
\cline { 2 - 6 } & Changed & Changed & Unchanged & Sum & Accuracy & Omission \\
SHP based & Unchanged & 1760 & 946 & 41766 & $98 \%$ & $2 \%$ \\
Change & Sum & 42600 & 59054 & 50834 & $97 \%$ & $3 \%$ \\
Detection & Accuracy & $96 \%$ & $98 \%$ & 92600 & \\
& Omission & $4 \%$ & $2 \%$ & & & \\
\cline { 2 - 7 } & Overall accuracy & $97 \%$ & & Kappa $=0.94$ & \\
\hline
\end{tabular}

Table 4. Accuracy of the proposed shape based change detection

The quantitative evaluation demonstrates that, with the utilization of the proposed method, the overall accuracy is slightly increased from $94 \%$ to $97 \%$. Also, Kappa coefficient is improved from 0.89 to 0.94 which reflect a higher degree of agreement between the result of the proposed method and ground truth shape as shown in the figure below:

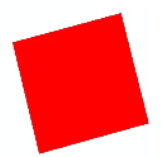

(a) Ground truth demolished building

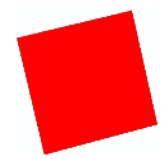

(b) The obtained demolished building by the proposed method

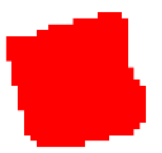

(c) The obtained demolished building by post-classification change detection
Figure 7. The obtained Demolished building.

When we look up the results in details, due to the exploitation of the images without resampling and with polygonal approximation of buildings shape, buildings change detection by shape matching for multi-resolution image produces a reliable change detection map comparing to post-classification change detection with image resampling.

\section{CONCLUSION}

Since urban area is the most changed landscape due to human activities and natural conditions, buildings change detection using remotely sensed images is the appropriate tool for urban evolution monitoring or damage estimation for crisis management.

For this purpose, the proposed method shown that shape matching is an effective tool to identify new and demolished buildings using multi-resolution satellite images without considering different pixel size effect. In addition, the use of the RMS value as a threshold to identify matched polygons allows avoiding strict image registration requirement for change detection process.

However, the developed method still limited to detect partial change of buildings and invalid to identify the nature of change (From-To). Thus, further work must consider the subsequent points: (1) Verify the efficiency of the proposed method with a large urban context (2) Identify 'From-To' change for new and demolished buildings (3) Extract buildings partial changes.

\section{ACKNOWLEDGMENT:}

This work was partly supported by National Natural Science Foundation of China under Grant Nos. 41622107 and
41371344, and Natural Science Foundation of Hubei Province in China under Grant No. 2016CFA029.

\section{REFERENCES}

Elsharkawy, A., Elhabiby M., El-Sheimy, N., 2012. New combined pixel/object-based technique for efficient urban classification using worldview-2 data. In: ISPRS Annals of the Photogrammetry, Remote Sensing and Spatial Information Sciences, Melbourne, Australia, Vol. XXXIX-B7, pp. 191-195.

Gang, C., Geoffrey, J. H., Luis, M. T. C., Michael, A. W., 2012. Object-based change detection. International Journal of Remote Sensing, 33 (14), pp. 4434-4457.

Lei, M., Manchun, L., Thomas, B., Xiaoxue, M., Dirk, T., Liang, C., Zhenjie C., Dong C., 2016. Object-Based Change Detection in Urban Areas: The Effects of Segmentation Strategy, Scale, and Feature Space on Unsupervised Methods. International Journal of Remote Sensing, 8 (9), pp. 1-18.

Penglin, Z., Zhiyong, L., Dan, Z., Jiangping, C., 2012. A shape similarity based change detection approach of Multi-resolution remote sensing images. In: ISPRS Annals of the Photogrammetry, Remote Sensing and Spatial Information Sciences, Melbourne, Australia, Vol. I-7, pp. 263-266.

Rene, C., Joanna, A., Jose, R., Alma, D., Maria, T., Pedro, A., Maria, I., Rainer, R., 2012. Potential effects in multi-resolution post-classification change detection. International journal of Remote Sensing, 33 (20), pp. 6426-6445.

Shabnam, J., Yun, Z., 2013. Very High Resolution Satellite Image Classification Using Fuzzy Rule-Based Systems. Algorithms, 6, pp. 762-781.

Yonghong, J., Mingting Z., Ye J., 2016. Object-oriented change detection based on multi-scale approach. In: The International Archives of the Photogrammetry, Remote Sensing and Spatial Information Sciences, Prague, Czech Republic, Vol. XLI-B7, pp. 517-522.

Zhang, S., Xu, L., 2008. The comparative study of three methods of remote sensing image change detection. In: The International Archives of the Photogrammetry, Remote Sensing and Spatial Information Sciences, Beijing, China, Vol. XXXVII, Part B7, pp. 1595-1598.

Zuoquan, Z., Roger, R. S., 2005. Measuring Similarity Among Various Shapes Based on Geometric Matching. Geographical Analysis, 37, pp. 410-422. 